\title{
Granulomatosis with Polyangiitis
}

National Cancer Institute

\section{Source}

National Cancer Institute. Granulomatosis with Polyangilitis. NCI Thesaurus. Code C3444.

A rare, autoimmune, systemic medium and small size vasculitis. It is characterized by the formation of necrotizing granulomas in the respiratory tract, necrotizing ang iitis, and glomerulonephritis. 